\title{
PRODUÇÃO DE MUDAS DO TIPO REBENTÃO, UTILIZANDO COROAS DE TRÊS CULTIVARES DE ABACAXI INOCULADAS COM FUNGOS MICORRÍZICOS ${ }^{1}$
}

\author{
PAULO CESAR DOS SANTOS 2 , SILVIO DE JESUS FREITAS 3 , \\ MARTA SIMONE MENDONÇA FREITAS ${ }^{4}$, LILIANE BARROSO DE SOUSA ${ }^{5}$, \\ ALMY JUNIOR CORDEIRO DE CARVALHO ${ }^{6}$
}

\begin{abstract}
RESUMO -Um dos principais gargalos para o desenvolvimento da abacaxicultura no Brasil tem sido a ausência tanto de mudas em quantidade quanto em qualidade, para propagação. Entre as alternativas, verifica-se a obtenção de mudas a partir da brotação de gemas de coroas dos frutos, que são, normalmente, descartadas pelo consumidor. Além disso, a utilização de fungos micorrízicos arbusculares (FMAs) pode ser uma alternativa para melhorar a produção das mudas, visto que estes fungos podem abreviar o tempo de formação de mudas de diversas frutíferas. O objetivo deste trabalho foi avaliar a produção de mudas do tipo rebentão, através do método de destruição do meristema apical da coroa de cultivares do abacaxizeiro inoculadas com FMAs. Utilizou-se o delineamento em blocos casualizados, num fatorial 3x3, com três cultivares de abacaxi ('Smooth Cayenne', 'Pérola' e 'Jupi') e três tratamentos microbiológicos (Sem inoculação, inoculação com Glomus etunicatum e inoculação com uma mistura dos fungos Glomus clarum e Gigaspora margarita), com quatro repetições. As primeiras emissões foram registradas aos 30; 60 e 90 dias após o plantio para as cultivares 'Smooth Cayenne', 'Pérola' e 'Jupi', respectivamente. O abacaxizeiro 'Smooth Cayenne' produziu 80 e 69\% de mudas a mais do que as cultivares 'Pérola' e 'Jupi', respectivamente. Coroas de abacaxi inoculadas com a mistura de fungos micorrízicos apresentaram maior número de emissão de brotações quando comparadas com aquelas inoculadas apenas com G. etunicatum. Nas avaliações nutricionais das coroas, a inoculação com a mistura de dois fungos micorrizicos promoveu, em folhas da coroa do abacaxizeiro, incrementos de 85 e $66 \%$ nos teores de P; de 22 e $13 \%$ para os de N, e de 6 e $19 \%$ para os de K, em relação aos tratamentos G. etunicatum e sem inoculação, respectivamente. Conclui-se que a produção de rebentos oriundos de coroas cuja gema principal foi decapitada é uma alternativa para a produção de mudas de abacaxizeiro, sendo mais eficiente na cultivar 'Smooth Cayenne', que produziu 26 mudas em coroas cultivadas até 420 dias após o plantio.
\end{abstract}

Termos para Indexação: Ananas comosus, micorriza, coroa, brotação.

\section{PRODUCTION OF SEEDLINGS OF TYPE SUCKERS, USING CROWS OF THREE CULTIVARS OF PINEPLE INOCULATED WITH MYCORRHIZAL FUNGI}

\begin{abstract}
One of the main obstacles to the development of pineapple cultivation in Brazil has been the absence of seedlings in both quantity and quality, for propagation. Among the alternatives, the obtaining of seedlings is verified starting from the sprouting from crowns of fruits, which are normally discarded by the consumer. Furthermore, the use of arbuscular mycorrhizal fungi (AMF) can be an alternative for improving the production of seedlings, since these fungi can shorten the time of seedlings of various fruit. In that sense, the objective of this study was to evaluate the production of seedlings type suckers, using the method of destruction of the apical meristem of the crown of the pineapple plants inoculated with FMAs. For such, it was used the randomized complete block design, factorial $3 \times 3$, with three cultivars of pineapple ('Smooth Cayenne', 'Pérola' and 'Jupi') and three microbiological treatments (no inoculation, Glomus etunicatum and a mixture of Glomus clarum and Gigaspora margarita), with four replications. The first emissions were recorded at 30,60 and 90 days after planting for cultivar Smooth, 'Pérola' and 'Jupi', respectively. The pineapple 'Smooth Cayenne' produced 80 to $69 \%$ seedlings more than the cultivars 'Pérola' and 'Jupí', respectively. In microbiological treatments there was a significant difference for the suckers issued, in which the mixed treatment was the most prevalent issue when compared with treatment with G. etunicatum. Nutritional assessments in the crowns, inoculation with the mixed treatment promoted an increase of 85 and $66 \%$ for P, 22 and $13 \%$ for N and 6 and $19 \%$ for K, compared to treatments G. etunicatum and control respectively. It is concluded that the production of suckers from crown whose principal gem was beheaded is an alternative for the production of pineapple seedlings, being more efficient in cultivar 'Smooth Cayenne' producing 26 seedlings at 420 days after planting.
\end{abstract}

Index terms: Ananas comosus, mycorrhiza, sprouting, crown.

\footnotetext{
1(Trabalho 176-10). Recebido em: 30-07-2010. Aceito para publicação em: 06-05-2011.

${ }^{2}$ Mestrando em Produção Vegetal, UENF/CCTA/LFIT, 28013-603, Campos-RJ, pcsantos18@hotmail.com

${ }^{3}$ DSc em Produção Vegetal, UENF/CCTA/LFIT, 28013-603, Campos-RJ, freitassj@yahoo.com.br

${ }^{4}$ DSc em Produção Vegetal, UENF/CCTA/LSOL, 28013-603, Campos-RJ, msimone@uenf.br

${ }^{5}$ Graduanda em Agronomia. UENF/CCTA/LFIT, 28013-603, Campos-RJ, lilianeuenf@hotmail.com

${ }^{6} \mathrm{DSc}$ em Produção Vegetal, Professor de Fruticultura, UENF/CCTA/LFIT, 28013-602, Campos-RJ, almy@uenf.br
} 


\section{INTRODUÇÃO}

O abacaxizeiro (Ananas comosus) é uma das mais importantes fruteiras plantadas no Brasil, sendo cultivada em todas as regiões, com produção estimada, em 2009, de 3.275.748 toneladas em uma área colhida de 61.024 ha (AGRIANUL 2010), sendo a sétima fruteira mais cultivada no País. As cultivares de abacaxi mais plantadas atualmente no Brasil são 'Pérola' e 'Smooth Cayenne', ambas suscetíveis à fusariose, principal problema fitossanitário da cultura no País, a qual é disseminada principalmente por meio de mudas de baixa qualidade.

No Brasil, o estágio atual do processo de produção de mudas do abacaxizeiro tem sido caracterizado especialmente pela ausência de material de qualidade, notadamente no que diz respeito à sanidade, e pela baixa oferta, com ausência de um sistema de produção profissionalizado e com valor unitário pouco competitivo para esta atividade. Ressalta-se, ainda, que comercialmente a totalidade de mudas de abacaxi no Brasil é originária de processo assexuado.

A carência da oferta de mudas de qualidade pode ser atribuída à baixa eficiência dos métodos, ao tempo relativamente longo para a obtenção das mudas em relação ao ciclo da cultura e ao alto custo de produção, elevando o preço final. Dessa forma, tornam-se imprescindíveis a melhoria e o desenvolvimento de técnicas de multiplicação, que tenham maior eficiência, rapidez e, principalmente, simplicidade na execução (COELHO et al., 2007).

Pesquisas têm desenvolvido métodos de propagação mais adequados que proporcionam a obtenção de mudas sadias, de boa qualidade e em quantidade suficiente para formação de novas lavouras.

A propagação empregando-se a coroa de abacaxi, não pelo plantio direto como muda, mas com a destruição mecânica de seu ápice caulinar, para indução de brotação das gemas laterais, foi apontada por Coelho et al. (2007) como alternativa para produção de mudas do tipo rebentão. Uma coroa, dependendo do seu tamanho, pode apresentar até 40 folhas, podendo desenvolver dezenas de gemas, que, por sua vez, poderão gerar vários rebentos.

Coelho et al. (2007) demonstraram o potencial das coroas na produção de mudas precoces de rebentos pelo método de destruição do meristema apical e produziram, em média, 5,2 mudas com 35 $\mathrm{cm}$ de comprimento, em 360 dias, e indicam, ainda, a possibilidade de que o número de rebentos emitidos e colhidos possam aumentar com a retirada constante do maior rebento, a intervalos mais curtos. Porém, o tamanho mínimo para a colheita ainda precisa ser definido, o que deve ser feito em função do destino que se daria aos rebentos, se para o plantio direto no campo ou para o transplantio no viveiro.

A introdução de fungos micorrízicos arbusculares (FMAs) tem um grande potencial nos programas de produção de mudas, pois permite abreviar o tempo de formação da muda de várias fruteiras, como mamão (MARTINS et al., 2000), maracujazeiro-doce (ANJOS et al., 2005), cajueiro (WEBER et al., 2004) e bananeira (LINS et al., 2003).

As micorrizas são amplamente reconhecidas pelo efeito positivo que proporcionam no crescimento da planta, pela melhoria na absorção de nutrientes, expandindo a zona de absorção da raiz, favorecendo a maior absorção de nutrientes como fósforo (FREITAS et al., 2006), nitrogênio e potássio (GUPTA et al., 2002). Em condições controladas, Marschner e Dell (1994) verificaram que os fungos micorrízicos podem ser responsáveis pela absorção de cerca de $80 \%$ de $\mathrm{P}, 25 \%$ do $\mathrm{N}$ e $10 \%$ de $\mathrm{K}$.

Faria et al. (2008) avaliaram os teores nutricionais e a porcentagem de colonização micorrízica em abacaxizeiro 'Smooth Cayenne' em função do tipo da muda (coroa, filhote e rebentão) e de dose de nitrogênio (4 e $15 \mathrm{~g}$ planta $^{-1}$ ciclo $^{-1}$ ), verificaram que o aumento nas doses de nitrogênio proporcionou decréscimo na colonização micorrízica e observaram, também, que a maior porcentagem de colonização micorrízica foi encontrada em mudas do tipo filhote, e a menor, no tipo coroa.

Gutierrez-Oliva et al. (2009), testando o efeito de dois FMAs com diferentes concentrações de fósforo na sobrevivência e crescimento de mudas de abacaxi micropropagadas, verificaram que os tratamentos não influenciaram na sobrevivência das mudas; no entanto, as mudas produziram maior número de folhas e peso seco quando foram inoculadas com o Glomus claroideum.

A utilização de FMAs na produção de mudas pode proporcionar aumento na produtividade do viveiro, na rotatividade da ocupação da infraestrutura e na eficiência de utilização de mão de obra especializada, além de melhorar a qualidade das mudas formadas.

A partir das informações expostas, o presente trabalho teve como objetivo verificar a produção de mudas do tipo rebentão, através do método de eliminação do ápice caulinar, de três cultivares de abacaxizeiro inoculadas com FMAs. 


\section{MATERIAL E MÉTODOS}

$\mathrm{O}$ experimento foi conduzido em casa de vegetação no Câmpus da Universidade Estadual do Norte Fluminense Darcy Ribeiro - UENF, no setor de Horticultura - Laboratório de Fitotecnia - CCTA, no período de 12-04-2008 a 12-07-2009. O delineamento experimental adotado foi o de blocos ao acaso (DBC) em sistema fatorial $3 \times 3$, sendo três cultivares de abacaxi ('Smooth Cayenne', 'Pérola' e 'Jupi') e três tratamentos microbiológicos (sem inoculação, inoculação com Glomus etunicatum e inoculação com uma mistura dos fungos Glomus clarum e Gigaspora margarita), com quatro repetições para cada tratamento.

As coroas foram destacadas dos frutos e selecionas por massa fresca: 'Smooth Cayenne' (150 - 200 g), 'Pérola' (100 - 150 g) e 'Jupi' $(60-90$ g), em seguida foram tratadas com solução de $0,5 \%$ de hipoclorito de sódio, durante 15 minutos, e depois lavadas com água desionizada. As coroas tiveram seu meristema apical eliminado manualmente, com auxílio de um alicate com ponta fina e uma chave de fenda.

Os inóculos foram adquiridos da coleção do Setor de Microbiologia do Laboratório de Solos da UENF. A multiplicação dos fungos ocorreu em casa de vegetação, utilizando-se como substrato de uma mistura de solo e areia na proporção de $1: 1(\mathrm{v} / \mathrm{v})$, respectivamente, autoclavados por duas vezes, a uma temperatura de $121^{\circ} \mathrm{C}$ por 1 hora. Utilizaram-se vasos com capacidade de $3 \mathrm{dm}^{3}$, onde foram adicionados o substrato e $50 \mathrm{~g}$ de inóculo (mistura de solo contendo esporos, hifas e raízes colonizadas) das espécies de fungos a serem estudadas. Em seguida, 10 sementes de milho (Zea mays) foram semeadas em cada vaso, sendo que, antes do plantio, as sementes foram desinfestadas com solução de $0,5 \%$ de hipoclorito de sódio, durante 10 minutos, e depois lavadas com água desionizada.

Quatro meses após o plantio do milho, a parte aérea foi cortada e, trinta dias após o corte, a mistura do solo contendo esporos, hifas e raízes finas foi utilizada como inóculo nos tratamentos do experimento.

O substrato utilizado para o plantio das coroas foi uma mistura de terra de superfície, areia lavada e de substrato Plantmax ${ }^{\circledR}$ hortaliça, nas proporções de 4:2:1 (v/v), respectivamente. Posteriormente, este substrato foi esterilizado em autoclave por duas vezes, a uma temperatura de $121^{\circ} \mathrm{C}$ por 1 hora, com a finalidade de eliminação dos FMAs nativos. Após a autoclavagem, o substrato apresentou as seguintes características químicas (Tabela 1).
A inoculação das mudas foi realizada no momento do plantio das coroas. O experimento foi conduzido em vasos de $5 \mathrm{dm}^{3}$, onde foram adicionados o substrato e $50 \mathrm{~g}$ do inóculo (mistura do solo contendo esporos, hifas e raízes finas cortadas) de 2 a $3 \mathrm{~cm}$ abaixo da superfície do substrato, de acordo com os tratamentos. As avaliações ocorreram num período de 420 dias após o plantio das coroas de abacaxi.

Semanalmente, quantificou-se o número de gemas intumescidas. Os rebentos foram colhidos quando atingiam cerca de $10 \mathrm{~cm}$, sendo avaliados: peso fresco, diâmetro e número de folhas. Ao final do experimento, realizou-se a contagem de gemas dormentes no caule e a análise nutricional dos macronutrientes da parte aérea e a colonização micorrízica das coroas decapitadas.

Aos 420 dias após o plantio, as coroas de abacaxi foram colhidas e colocadas para secar em estufa com temperatura controlada a $70^{\circ} \mathrm{C}$, em seguida foram moídas em moinho tipo Wiley, com peneira de 20 mesh, e armazenadas em frascos hermeticamente fechados. Para a determinação dos nutrientes do material moído, foram pesadas duas amostras de cada tratamento para se proceder, respectivamente, às digestões sulfúricas e nitroperclórica. Os teores de nitrogênio foram determinados pela digestão sulfúrica, enquanto os teores de $\mathrm{P}, \mathrm{K}, \mathrm{Ca}$ e $\mathrm{Mg}$ foram determinados pela digestão nitroperclórica.

$\mathrm{O} \mathrm{K}$ foi dosado por espectrofotometria de emissão atômica; o $\mathrm{P}$, determinado colorimetricamente, pelo método do molibdato; o $\mathrm{Ca}$ e $\mathrm{Mg}$, por espectrometria de absorção atômica. O N orgânico foi dosado pelo método de Nessler (JACKSON, 1965).

Para a avaliação da infecção micorrízica, as raízes foram removidas dos vasos, lavadas com água corrente. As raízes finas foram armazenadas em álcool etílico a 50\% para proceder à avaliação da porcentagem de colonização radicular. Para isto, as raízes foram coloridas segundo Grace e Stribley (1991) com as seguintes adaptações: clareamento das raízes por 5 minutos a $80^{\circ} \mathrm{C} \operatorname{com~} \mathrm{KOH}$ a $2,5 \%$, e posterior coloração por 20 minutos a $80^{\circ} \mathrm{C}$ com azul de metil. Em seguida, as raízes foram levadas ao microscópio estereoscópico com aumento de $20 \mathrm{X}$ para determinar o grau de infecção.

\section{RESULTADOS E DISCUSSÃO}

$\mathrm{Na}$ análise de colonização do sistema radicular, verificou-se que todos os tratamentos microbiológicos (exceto o controle) foram capazes de se associar às raízes do abacaxizeiro. $\mathrm{O}$ inóculo misto colonizou todas as cultivares, não havendo diferença 
estatística entre elas. Diferentemente foi verificado com o inóculo G. etunicatum, onde este apresentou maior índice de colonização na cultivar 'Pérola', quando comparado com as cultivares 'Smooth Cayenne' e 'Jupi', que foram estatisticamente semelhantes. Para todas as cultivares avaliadas, o inóculo misto colonizou mais que o G. etunicatum (Tabela 2).

Os primeiros rebentos foram emitidos aos 30; 60 e 90 dias após o plantio das coroas de abacaxi para as cultivares 'Smooth Cayenne', 'Pérola' e 'Jupi', respectivamente. Coelho et al. (2007), empregando a decapitação mecânica em coroas de abacaxizeiro 'Smooth Cayenne', verificaram as primeiras emissões aos 33 dias.

Quanto ao número total de rebentos emitidos aos 420 dias após o plantio da coroa de abacaxi, a cultivar 'Smooth Cayenne' mostrou-se superior às demais (Tabela 3). Esta cultivar naturalmente produz mais mudas do tipo rebento, o que pode explicar a diferença no número de mudas produzidas entre as cultivares. Castro e Kluge (1998) relatam que a cultivar 'Smooth Cayenne', por possuir um talo mais volumoso e com maior número de gema axilares, normalmente produz mais mudas do tipo rebentão do que a cultivar 'Pérola'.

Verificou-se efeito do tratamento microbiológico sobre o número de rebentos emitidos (Tabela 3). A utilização do inóculo misto proporcionou maior emissão de rebentos quando comparado com o tratamento com G. etunicatum.

Marschner e Dell (1994) afirmaram que, normalmente a eficiência micorrízica está relacionada com a quantidade de micélio externo formado no solo. Certos fungos podem ter grande capacidade de colonizar o hospedeiro, mas a proporção de hifas externas (estruturas que permitem a maior absorção de nutrientes) varia muito entre as espécies de FMAs.

Algumas associações fungo-hospedeiro, dependendo do genótipo da planta, da espécie do fungo e da disponibilidade de P no solo, podem proporcionar baixo crescimento vegetal, podendo chegar a atingir uma condição de parasitismo, onde o balanço energético se torna desfavorável à planta (MARSCHNER; DELL, 1994).

No decorrer do experimento, observou-se que novas brotações ocorriam somente quando se destacavam os rebentos (10 $\mathrm{cm}$ de comprimento), pois estes funcionavam como fonte temporária de auxina. Assim, com a eliminação do sítio de produção desse fito-hormônio, novas gemas eram induzidas a brotar, devido à diminuição da relação auxina/citocininas nos tecidos; através desta observação, constatou-se a inibição do desenvolvimento de novos rebentos pela presença de outro dominante.

A inoculação com o tratamento misto promoveu a redução do tempo em que os rebentos ficavam aderidos à planta-mãe, atingiam comprimento de 10 $\mathrm{cm}$ mais rapidamente, e consequentemente novas gemas eram induzidas a brotar, demonstrando o efeito clássico da dominância apical.

Jaizme-Vega e Azcón (1991) demonstraram que a produção de biomassa de mudas micropropagadas de abacaxizeiro aumentou em 142\% e 148\% com a inoculação de G. fasciculatum e G. mosseae, respectivamente. Jaizme-Vega e Azcón (1995) obtiveram um aumento de $322 \%$ e $294 \%$ de matéria seca da parte aérea para o abacaxizeiro infectado com $G$. mosseae e G. fasciculatum, respectivamente.

Coelho et al. (2007) verificaram em média 12,67 rebentos emitidos nas coroas de abacaxi da cultivar 'Smooth Cayenne' aos 420 dias após o plantio; no entanto, os rebentos eram colhidos quando estes atingiam aproximadamente $25-30 \mathrm{~cm}$. No presente trabalho, a cultivar 'Smooth Cayenne' emitiu, em média, 25,83 rebentos, sendo que estes foram destacados da coroa com cerca de $10 \mathrm{~cm}$ (Tabela 3 ), sendo necessário passar por um período de ceva no canteiro até atingirem o tamanho ideal para serem levados ao campo $(25-30 \mathrm{~cm})$.

$\mathrm{Na}$ avaliação dos rebentos colhidos, a cultivar 'Smooth Cayenne' foi superior em relação às demais, produzindo $80 \%$ e $69 \%$ a mais que as cultivares 'Pérola' e 'Jupi', respectivamente (Tabela 3). Coelho et al. (2007) colheram 5,2 mudas do abacaxizeiro 'Smooth Cayenne' com peso médio de $145 \mathrm{~g}$ e comprimento de $35,9 \mathrm{~cm}$ aos 360 dias após o plantio da coroa de abacaxi. O autor relatou que não existe encontrou nenhum resultado semelhante por outro método de multiplicação do abacaxizeiro, observado o período decorrido entre o plantio da matriz e a colheita dos primeiros rebentos.

As variáveis: diâmetro médio e número de folhas dos rebentos foram influenciadas pelas diferentes cultivares, sendo que o abacaxizeiro 'Smooth Cayenne' apresentou rebentos com menor média de diâmetro e com maior número de folhas, não sendo observada diferença significativa entre as cultivares 'Pérola' e 'Jupi'. Para o peso médio dos rebentos, a cultivar 'Pérola' foi estatisticamente superior a 'Smooth Cayenne'; no entanto, não diferiu das médias observadas na variedade 'Jupi' (Tabela 4).

Os tratamentos microbiológicos não proporcionaram diferença significativa sobre as variáveis: diâmetro médio, peso médio e número médio de folhas dos rebentos (Tabela 4).

Nas avaliações realizadas nas coroas de abacaxi, ao final do experimento, não houve interação 
significativa entre os tratamentos. No momento da implantação do experimento, as coroas do abacaxizeiro Smooth possuíam, em média, $50 \mathrm{~g}$ a mais do que as coroas do Pérola; apesar disso, ao final do experimento, as coroas do Smooth apresentaram menor peso seco, provavelmente pelo fato de as coroas de Smooth terem emitido mais rebentos, acarretando no maior consumo de suas reservas (Tabela 5). As coroas da variedade Jupi apresentaram menor média de matéria seca, pois estas tiveram menor massa fresca na instalação do experimento.

$\mathrm{Na}$ quantificação do número de gemas não brotadas, a cultivar 'Pérola' foi estatisticamente superior à 'Jupi', sendo que estas não diferiram da variedade 'Smooth Cayenne' (Tabela 5).

Quanto à avaliação nutricional das coroas de abacaxi, não foi verificado interação significativa entre os tratamentos. Avaliando isoladamente o fator cultivar, verificou-se que o teor de $\mathrm{N}$ e $\mathrm{Ca}$ seguiram a mesma tendência, onde as cultivares 'Smooth Cayenne' e 'Jupi' não diferiram significativamente; já o abacaxizeiro 'Pérola' apresentou os menores teores destes nutrientes. Para os teores de P, a cultivar 'Smooth Cayenne' apresentou teor superior ao da cultivar 'Jupi'. Os tratamentos não proporcionaram diferença significativa nos teores médios de $\mathrm{K}$ e $\mathrm{Mg}$ (Tabela 6). Quanto ao efeito dos FMAs na nutrição das coroas de abacaxi, verificou-se que a inoculação com o tratamento misto proporcionou teores de N, P e K superiores, com incremento de 85 e $66 \%$ para o P; 22 e $13 \%$ para o N, e 6 e $19 \%$ para o K, em relação aos tratamentos G. etunicatum e o controle, respectivamente (Tabela 6). Esses valores expressam a capacidade dos FMAs em promover o aumento na absorção de nutrientes do solo.

Vitorazi Filho (2008) verificou que a inoculação com Glomus clarum promoveu ganho de 86;129 e $108 \%$ no teor de N, K e Ca, respectivamente, presentes na matéria seca da parte aérea de plantas de carambola.

Jaizme-Vega e Azcón (1991) demonstraram que o conteúdo de NPK na parte aérea de mudas micropropagadas de abacaxizeiro aumentou, respectivamente, em $152 \%, 241 \%$ e $210 \%$ com o uso de G. fasciculatum e $270 \%, 123 \%$ e $192 \%$ com o uso de G. mosseae.

Freitas et al. (2006) observaram que a inoculação de plantas de menta com G. clarum e $G$. margarita elevaram os conteúdos de $\mathrm{K}$ na parte aérea em 139 e $142 \%$, respectivamente, quando comparado com os teores observados em plantas não inoculadas.

Trindade et al. (2001) observaram em mudas de mamoeiro do grupo solo e formosa que a inoculação com G. margarita proporcionou maiores teores e conteúdos de fósforo na parte aérea, quando comparados ao tratamento sem inoculação.

O tratamento microbiológico com o $G$. etunicatum não promoveu incremento na absorção de $\mathrm{N}$ e $\mathrm{P}$, não sendo indicado para as cultivares no

ambiente estudado.

TABELA 1 - Análise química do substrato após autoclavagem por duas vezes, a uma temperatura de $121^{\circ} \mathrm{C}$ por 1 hora.

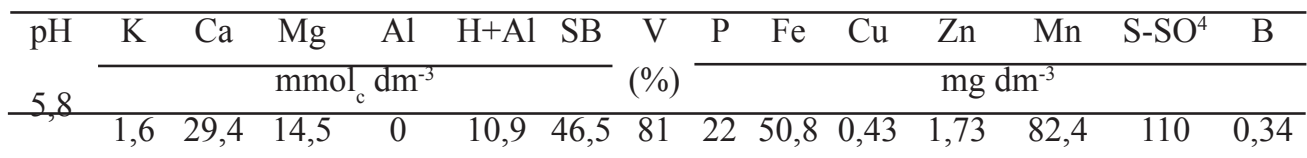

Matéria orgânica $=35,7 \mathrm{~g} \mathrm{dm}^{-3}$

Análises realizadas pelo Laboratório de Análise de Solos da Fundenor, Campos dos Goytacazes-RJ.

TABELA 2 - Colonização dos FMAs nas raízes de coroas de cultivares de abacaxizeiro, em função de tratamentos microbiológicos.

\begin{tabular}{ccccc}
\hline \multirow{2}{*}{ Cultivar } & \multicolumn{3}{c}{ Tratamento microbiológico } & \multirow{2}{*}{ Média } \\
& Misto & G. etunicatum & Sem fungo & \\
'Smooth' & $100 \mathrm{Aa}$ & $30 \mathrm{Bb}$ & $0 \mathrm{Ca}$ & 43,33 \\
'Pérola' & $95,0 \mathrm{Aa}$ & $80 \mathrm{Ba}$ & $0 \mathrm{Ca}$ & 58,33 \\
\hline 'Jupí' & $97,5 \mathrm{Aa}$ & $30 \mathrm{Bb}$ & $0 \mathrm{Ca}$ & 42,50 \\
\hline Média & 97,5 & 46,66 & 0 & \\
\hline CV (\%) & \multicolumn{5}{c}{15,44} & &
\end{tabular}


TABELA 3- Número total de rebentos emitidos e colhidos por coroa de cultivares de abacaxizeiro, em função de tratamentos microbiológicos, até 420 dias após o plantio.

\begin{tabular}{ccccc} 
& \multicolumn{3}{c}{ Tratamento microbiológico } & Média \\
\cline { 2 - 4 } Cultivar & Misto & G. etunicatum & Sem fungo \\
\hline Número total de rebentos emitidos \\
'Smooth' & 29,25 & 25,25 & 23,00 & $25,83 \mathrm{a}$ \\
'Pérola' & 16,75 & 13,00 & 16,75 & $15,50 \mathrm{~b}$ \\
'Jupí' & 15,50 & 12,25 & 13,75 & $13,83 \mathrm{~b}$ \\
\hline Média & $20,50 \mathrm{~A}$ & $16,83 \mathrm{~B}$ & $17,83 \mathrm{AB}$ \\
\hline CV (\%) & \multicolumn{5}{c}{17,31} \\
\hline \multicolumn{5}{c}{ Número total de rebentos colhidos } \\
'Smooth' & 25,75 & 24,25 & 21,25 & $23,75 \mathrm{a}$ \\
'Pérola' & 16,00 & 11,75 & 16,00 & $14,58 \mathrm{~b}$ \\
\hline 'Jupi' & 11,75 & 10,00 & 11,75 \\
\hline Média & $17,83 \mathrm{~A}$ & $15,33 \mathrm{~A}$ & $16,33 \mathrm{~A}$ \\
\hline CV (\%) & \multicolumn{5}{c}{15,39}
\end{tabular}

Médias seguidas de mesma letra maiúsculas nas linhas e minúscula nas colunas não diferem entre si pelo teste de Tukey $(\mathrm{P}>0,05)$.

TABELA 4 - Diâmetro médio dos rebentos (DMR), em mm, peso médio dos rebentos (PMR), em g, e número médio de folhas (NMF), quando colhidos com aproximadamente $10 \mathrm{~cm}$ de comprimento em função das cultivares e dos tratamentos microbiológicos.

\begin{tabular}{cccc}
\hline & DMR & PMR & NMF \\
\hline \multicolumn{4}{c}{ Cultivares } \\
\hline 'Smooth' & $16,51 \mathrm{~b}$ & $11,91 \mathrm{~b}$ & $11,73 \mathrm{a}$ \\
'Pérola' & $18,71 \mathrm{a}$ & $15,05 \mathrm{a}$ & $9,31 \mathrm{~b}$ \\
'Jupi' & $18,00 \mathrm{a}$ & $13,84 \mathrm{ab}$ & $9,11 \mathrm{~b}$ \\
\hline Média & 17,74 & 13,6 & 10,05 \\
\hline CV (\%) & 6,09 & 15,61 & 4,50 \\
\hline \multicolumn{4}{c}{ Tratamento microbiológico } \\
Misto & $17,39 \mathrm{a}$ & $13,72 \mathrm{a}$ & $10,30 \mathrm{a}$ \\
G. etunicatum & $18,30 \mathrm{a}$ & $13,77 \mathrm{a}$ & $10,00 \mathrm{a}$ \\
\hline Sem fungo & $17,53 \mathrm{a}$ & $13,31 \mathrm{a}$ & $9,85 \mathrm{a}$ \\
\hline Média & 17,74 & 13,6 & 10,05 \\
CV (\%) & 6,09 & 15,61 & 4,50 \\
\hline
\end{tabular}

Médias seguidas de mesma letra nas colunas não diferem entre si pelo teste de Tukey $(\mathrm{P}>0,05)$.

TABELA 5 - Peso seco da parte aérea da coroa de abacaxi (PSAC), em g, peso seco da raiz da coroa de abacaxi (PSRC), em g, e número de gemas dormentes na coroa de abacaxi (NGD), aos 420 dias após o plantio das coroas.

\begin{tabular}{cccc}
\hline Cultivar & PSAC & PSRC & NGD \\
\hline 'Pérola' & $38,28 \mathrm{a}$ & $2,62 \mathrm{a}$ & $8,25 \mathrm{a}$ \\
'Smooth' & $30,71 \mathrm{~b}$ & $2,04 \mathrm{ab}$ & $6,67 \mathrm{ab}$ \\
'Jupi' & $23,47 \mathrm{c}$ & $1,22 \mathrm{~b}$ & $5,91 \mathrm{~b}$ \\
\hline Média & 30,82 & 1,96 & 6,94 \\
\hline CV (\%) & 19,63 & 57,99 & 32,54 \\
\hline
\end{tabular}


Médias seguidas de mesma letra nas colunas não diferem entre si, pelo teste de Tukey $(P>0,05)$.

TABELA 6 - Teores dos nutrientes, em $\mathrm{g} \mathrm{kg}^{-1}$ da matéria seca, em coroa de cultivares de abacaxi em função das variedades e dos tratamentos microbiológicos.

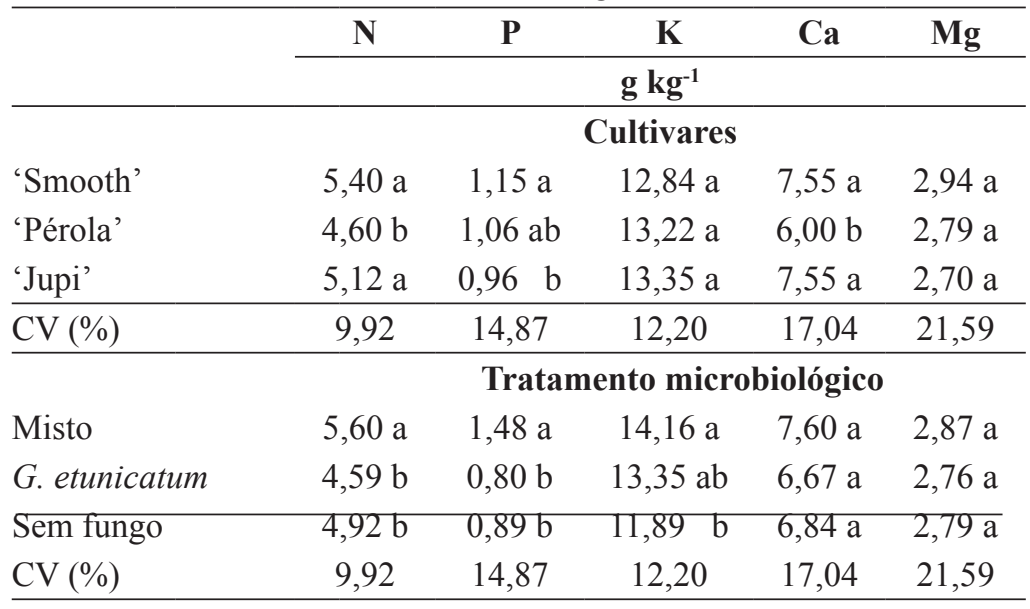

Médias seguidas de mesma letra nas colunas não diferem entre si, pelo teste de Tukey $(P>0,05)$.

\section{CONCLUSÕES}

1-Os resultados encontrados indicam que a coroa de abacaxi pode ser utilizada como material propagativo, notadamente no abacaxizeiro 'Smooth Cayenne', que produz, em média, 29,2 rebentos com cerca de $10 \mathrm{~cm}$ de comprimento, aos 420 dias após o plantio.

2-A utilização da mistura com duas espécies de fungos micorrízicos proporciona maior emissão de rebentos quando comparada com a utilização de apenas uma espécie de fungo.

3-Os fungos micorrízicos não influenciam no número de rebentos colhidos e nas avaliações biométricas destes. Entretanto, na análise nutricional das coroas de abacaxi, o tratamento microbiológico com a mistura dos fungos proporciona incremento significativo nos teores de nitrogênio e fósforo.

\section{REFERÊNCIAS}

AGRIANUAL: Anuário da agricultura brasileira. São Paulo: Instituto FNP, 2010. 504p.

ANJOS, E.C.T.; CAVALCANTE, U.M.T.; SANTOS, V.F.; MAIA, L.C. Produção de mudas de maracujazeiro-doce micorrizadas em solo desinfestado e adubado com fósforo. Pesquisa Agropecuária Brasileira, Brasília, v.40, n.4, p.345-351, 2005.

CASTRO, P.R.C.; KLUGE, R.A. Ecofisiologia de fruteiras tropicais. São Paulo: Nobel, 1998. 111 p.
COELHO, R.I.; CARVALHO, A.J.C. DE; LOPES, J.C.; TEIXEIRA, S.L.; MARINHO, C.S. Coroa do abacaxi 'Smooth Cayenne' na produção de mudas do tipo rebentão. Ciência e Agrotecnologia, Lavras, MG, v.31, p.1867-1871, 2007.

FARIA, D.C.; CARVALHO, A.J.C. DE; FREITAS, S.J. DE; FREITAS, M.S.M.; MARTINS, M.A.; SILVA, C.F. Diversidade de fungos micorrízicos arbusculares em mudas de abacaxizeiro cultivadas no campo, sob doses de nitrogênio. In: REUNIÃO ANUAL DA SOCIEDADE INTERAMERICANA DE HORTICULTURA TROPICAL, 52., 2008, Vitória. Resumos... Fortaleza: Embrapa Agroindústria Tropical, 2008. p.167-170.

FREITAS, M.S.M.; MARTINS, M.A.; CARVALHO, A.J.C. DE. Crescimento e composição da menta em resposta à inoculação com fungos micorrízicos arbusculares e adubação fosfatada. Horticultura Brasileira, Brasília, n.24, p.11-16, 2006.

GRACE, C.; STRIBLEY, P. A afer procedure fot routine staining of vesicular-arbuscular mycorrhizal fungi. Mycological Research, Cambridge, v.95, p.1160-1162, 1991.

GUPTA M.L.; PRASAD, A.; RAM, M.; KUMAR, S. Effect of vesicular-arbuscular mycorrhizal (VAM) fungus Glomus fasciculatum on the essential oil yield related characters and nutrient acquisition in the crops of different cultivars of menthol mint (Mentha arvensis) under field conditions. Bioresource Technology, Essex, v.81, p.77-79, 2002. 
GUTIÉRREZ-OLIVA, V.F.; ABUD-ARCHILA, M.F.A.; ALVAREZ, S.J.D.; GUTIÉRREZ-MICELI, F.A. Influencia de los hongos micorrizicos arbusculares sobre el crecimiento de vitro plantulas de pina (Ananas comosus (1.) Merr.) Con diferentes niveles de fosforo. Gayana Botanica, Concepción, n.66, p.1-9, 2009.

JACKSON, M.L. Soil chemical analysis. New Jersey: Prentice Hall, 1965. 498 p.

JAIZME-VEGA, M.C.; AZCÓN, R. Effect of vesicular-arbuscular mycorrhizal fungi on pineapple [Ananas comosus (L.)Merr.]. Fruits, Paris, n.46, p.47-50, 1991.

JAIZME-VEGA, M.C.J.; AZCÓN, E.R. Responses of some tropical and subtropical cultures to endomycorrhizal fungi. Mycorrhiza, Berlin, n.5, p.213-217, 1995.

LINS, G.M. DE L.; TRINDADE, A.V.; ROCHA, H.S. Utilização de Gigaspora margarita em plantas micropropagadas de bananeira em diferentes estádios de enraizamento. Revista Brasileira de Fruticultura, Jaboticabal, v.25, n.1, p.143-147, 2003.

MARSCHNER, H.; DELL, B. Nutrient uptake in mycorrhizal symbiosis. Plant and Soil, Dordrecht, n.159, p.89-102, 1994.
MARTINS, M.A.; GONÇALVES, G.F.; SOARES, A.C.F. Efeito de fungos micorrízicos arbusculares associados a compostos fenólicos, no crescimento de mudas de mamoeiro. Pesquisa Agropecuária Brasileira, Brasília, v.35, n.7, p.1465-1471, 2000.

TRINDADE, A.V.; DANTAS, J.L.L.; ALMEIDA, F.P.; MAIA, I.C.S. Estimativa do coeficiente de determinação genotípica em mamoeiros (Carica papaya L.) inoculados com fungo micorrízico arbuscular. Revista Brasileira de Fruticultura, Jaboticabal, v.23, n.3, p.607-612, 2001.

VITORAZI FILHO, J.A. Fungos Micorrízicos Arbusculares e adubação fosfatada na produção de mudas de caramboleira. 2008. 37 f. Monografia (Graduação em Agronomia) - Universidade Estadual do Norte Fluminense Darcy Ribeiro, Campos dos Goytacazes, 2008.

WEBER, O.B.; SOUZA, C.C.M.; GONDIN, D.M.F.; OLIVEIRA, F.N.S.; CRISÓSTOMO, L.A.; CAPRON, A.L.; SAGGIN JÚNIOR, O. Inoculação de fungos micorrízicos arbusculares e adubação fosfatada em mudas de cajueiro-anão-precoce. Pesquisa Agropecuária Brasileira, Brasília, v.39, p.477-483, 2004. 